\section{Timing and modality of the sclerosing agents binding to the human proteins: laboratory analysis and clinical evidences}

\author{
Lorenzo Tessari, ${ }^{1}$ Marcello Izzo, ${ }^{2}$ \\ Attilio Cavezzi, ${ }^{3}$ Francesco Zini, ${ }^{4}$ \\ Mirko Tessari, 5 Mario Ambrosino, 6 \\ Roberto Fanelli ${ }^{7}$
}

${ }^{1}$ Glauco Bassi Foundation, Trieste; ${ }^{2}$ Math.

Tech. Med., University of Ferrara;

${ }^{3}$ Eurocenter Venalinfa, San Benedetto del

Tronto (AP); ${ }^{4}$ Casa di Cura Città di

Parma, Parma; ${ }^{5}$ Vascular Diseases Center,

University of Ferrara; ${ }^{\circ}$ Centro Duomo

Analisi, Nola (NA); ${ }^{7}$ Istituto

Farmacologico Mario Negri, Milano, Italy

\section{Abstract}

Sclerosing agents (SA) are blood inactivated. Nevertheless, investigations concerning the interaction among SA and blood components have never been deeply investigated. Aim of the study is to precisely identify SA blood ligands, to determine their binding time and to highlight the clinical consequences. Thirty-one blood samples were collected from chronic venous disease patients and tested by capillary and agarose gel (AGE) electrophoresis before and after adding polidocanol (POL) and sodiumtetradecylsulphate (STS). The two different types of electrophoresis allowed an evaluation of the blood proteins binding with the sclerosing agents, with a reaction time lower than 8 seconds for the AGE. Subsequently six patients underwent foam sclerotherapy and then were subdivided in group A (4 patients) and B (2 patients). In group A blood sample was obtained from the ipsilateral brachial vein immediately before (T0) and repeated $1,3,5$, and 10 minutes after injection of STS 3\% injection into the GSV. In group B, the same procedure was performed with the same timing from the ipsilateral femoral vein. Free STS (fSTS) and total proteinbound STS (bSTS) were measured. POL mainly binds to $\beta$-globulins (11\%), while STS to albumin and $\alpha$-globulins (62.6\% and $30.7 \%)$ on the protidogram, respectively. Both in the brachial and in the femoral vein, the average fSTS was always 0 . STS binds to albumin (62.6\%) and $\alpha$ globulins (30.7\%), while POL is bound mainly by the $\beta$-globulins (11\%). The present paper demonstrates how the vast majority of the sclerosing agent is bound to the blood proteins, suggesting the need to look for possible sclerotherapy complications factors also in the used gas and/or in the subsequent cathabolites release.

\section{Introduction}

Foam sclerotherapy (FS) has become an important tool in chronic venous disease (CVD) treatment. ${ }^{1}$

Worldwide, the two most commonly used sclerosing agents (SA) in foam production are sodium tetradecyl sulphate (STS) and polidocanol (POL)

A few very interesting papers evaluated the SA in vitro effects on coagulation, ${ }^{2,3}$ together with the same SA inactivation by plasma protein. ${ }^{4}$

The STS deactivation by the blood was precisely assessed in vitro, demonstrating how small blood volumes can totally inactivate the sclerosing drug. Nevertheless, this fundamental investigation was performed only on bovine samples. ${ }^{5}$

In everyday clinical practice, FS has been demonstrated to be a safe and effective therapeutic option ${ }^{6,7}$ that has been made even safer by the recent use of carbon dioxide-oxygen in place of atmospheric air. ${ }^{8-10}$

Nevertheless, up to now, investigations concerning the precise interaction between the SA and the human blood components, once the drug reaches the vascular bed, are still lacking.

Moreover, the pathogenetic mechanisms underlying the origin of FS-induced complications remain unclear. ${ }^{11,12}$

The aim of this study is to precisely identify SA blood ligands, to determine their binding time and to highlight the associated clinical consequences.

\section{Materials and Methods}

Two parallel investigations were performed.

In the first one, 31 blood samples were collected following $12 \mathrm{~h}$ or fasting in CVD patients (C1-6 Ep As Pr) and tested both by capillary (CE) and agarose gel (AGE) electrophoresis, in order to obtain a normal control curve, to be subsequently compared after addition of sclerosing agents. The assessment was performed at 5, 8, 12, 14 and 16 min by CE evaluation and at $8 \mathrm{~s}$ by AGE. Subsequently, the same 31 patients blood samples were evaluated in new CE and AGE runs adding both 3\% STS and 3\% POL ( $200 \mu \mathrm{L}$ of SA+400 $\mu \mathrm{L}$ of blood). In CE the proteins fractions separations occurred in a fused silica capillary, which was previously filled with a buffer solution. The main components of the system are a vial with the sample, a source and a destination recipient, electrodes, a high voltage henerator, a shallow capillary (in silica), a recorder, a data collector and the support. The migration of the particle is created by the high voltage that was applied to
Correspondence: Lorenzo Tessari, via Giovanni Falcone 24/B, 37019 Peschiera del Garda (VR), Italy.

Tel.: +39.045.6401681 - Fax: +39.045 .6409147 .

E-mail: mirko@tessaristudi.it

Key words: sclerosing agents, blood proteins, sclerotherapy, safety.

Contributions: LT, study design, data collection, data analysis, critical review, final approval; MI, data analysis, critical review, final approval; AC, data collection, data analysis, writing, critical review, final approval; FZ, data collection, data analysis, final approval; MT, data collection, final approval; MA, data analysis, critical review, writing, final approval; RF, data analysis, critical review, writing, final approval.

Conflict of interests: the authors declare no potential conflict of interests.

Received for publication: 23 March 2014.

Revision received: 14 June 2014.

Accepted for publication: 18 June 2014

This work is licensed under a Creative Commons Attribution 3.0 License (by-nc 3.0).

(C) Copyright L. Tessari et al., 2014

Licensee PAGEPress, Italy

Veins and Lymphatics 2014; 3:3275

doi:10.4081/vl.2014.3275

the same solution, thus reducing the migration time to $5 \mathrm{~min}$. The proteins were then assessed and quantified by direct measurement of their $214 \mathrm{~nm}$ wave length absorbance. ${ }^{13-20}$

In AGE, proteins fractions separation is based on the competition between he applied electric field and the specific protein affinity to the media. In AGE, an agarose gel matrix is used to deposit the particles. Then the proteins separate because of their different charge.

The band quantification, together with the standard diagram production, occurs by swartz starch or other substances coloration. Subsequently, a cleaning and media diaphanization are performed. At the end of the processing, the colored bands film is created. A densitometer is used for the assessment. The particles separation is obtained following their different electrophoretic mobility. ${ }^{13-20}$

The study population mean age was $54 \pm 8$ years, with a female/male ratio of 5/1. No significant co-morbidities were present.

The binding time evaluation was also performed for the SA and the plasma proteins.

In the second investigation, six patients among the 31 who previously underwent the blood collection, were treated by injection of $3 \%$ (1 cc) STS FS in the GSV trunk, and then divided into group A (4 patients) and B (2 patients).

In group $\mathrm{A}$, a blood sample was obtained from the ipsilateral brachial vein, before an 
STS 3\% injection into the GSV (T0). The sampling was repeated $1,3,5,10$ min later.

In group B (2 patients), the same procedure was performed with the same timing on the ipsilateral femoral vein.

Free STS (fSTS) and total protein-bound STS (bSTS) were measured in both groups.

The foam was produced according to the Tessari technique, thus mixing 1 cc of $3 \%$ STS with 4 cc of gas. ${ }^{21}$

The blood samples were immediately spindried and the plasma was stored in another vial.

Everything was frozen at $26^{\circ}$ and subsequently sent to the laboratory.

fSTS and total protein-bSTS were measured.

In group B ( 2 patients, 1 male and 1 female), the same procedure was performed with the same timing on the ipsilateral femoral vein.

An informed consent was obtained from all the patients, both for the clinical and the laboratory procedures.

\section{Results}

All the set-up tests were correctly performed and reproduced reliable outcomes. All the proteins fractions absolute values were physiological (Figure 1).

In the first set of investigations, the human plasma proteins displayed a weak bond with POL, mainly in the $\beta 1$ an $\beta 2$ range (only about $11 \%$ of the plasma proteins pool) (Figure 2A).Conversely, the STS-human plasma protein interaction was especially represented in the albumin (62.6\%), $\alpha 1(10,7 \%)$ and $\alpha 2$ (20.0\%) fractions (Figure 2B).

In the binding time analysis, the CE evaluation at 5, 8, 12, 14 and 16 min highlighted a total overlap of STS 3\%-plasma proteins (1:2 ratio respectively) with the basal plasma proteins electrophoretic run (Figure 3).

The AGE evaluation demonstrated an 8-s drug-protein binding peak, that was equally maintained at the 12 and $60 \mathrm{~s}$ evaluation (Figure 4).

The second investigation, concerning
fSTS amount, demonstrated that in the brachial vein, the average bSTS concentration in $\mu \mathrm{g} / \mathrm{mL}$ was $0,0.568,5.98,6.91$ and 7.2 , respectively at $\mathrm{T} 0,1,3,5,10 \mathrm{~min}$.

In the femoral vein, bSTS average concentration in $\mathrm{g} / \mathrm{mL}$ was $0,1.62,13,24.6$ and 8.67, respectively at $\mathrm{T} 0,1,3,5,10 \mathrm{~min}$.

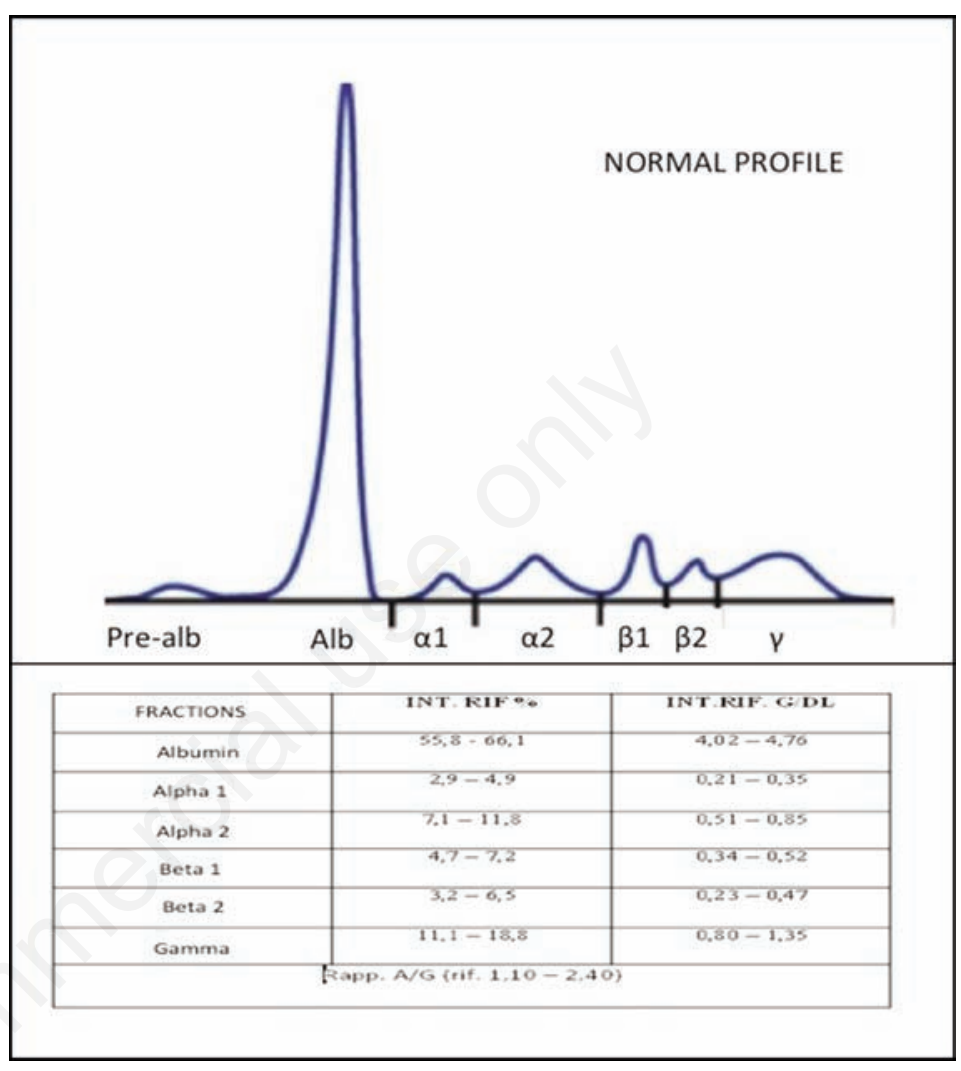

Figure 1. Normal electrophoretic run with proteins percentages.

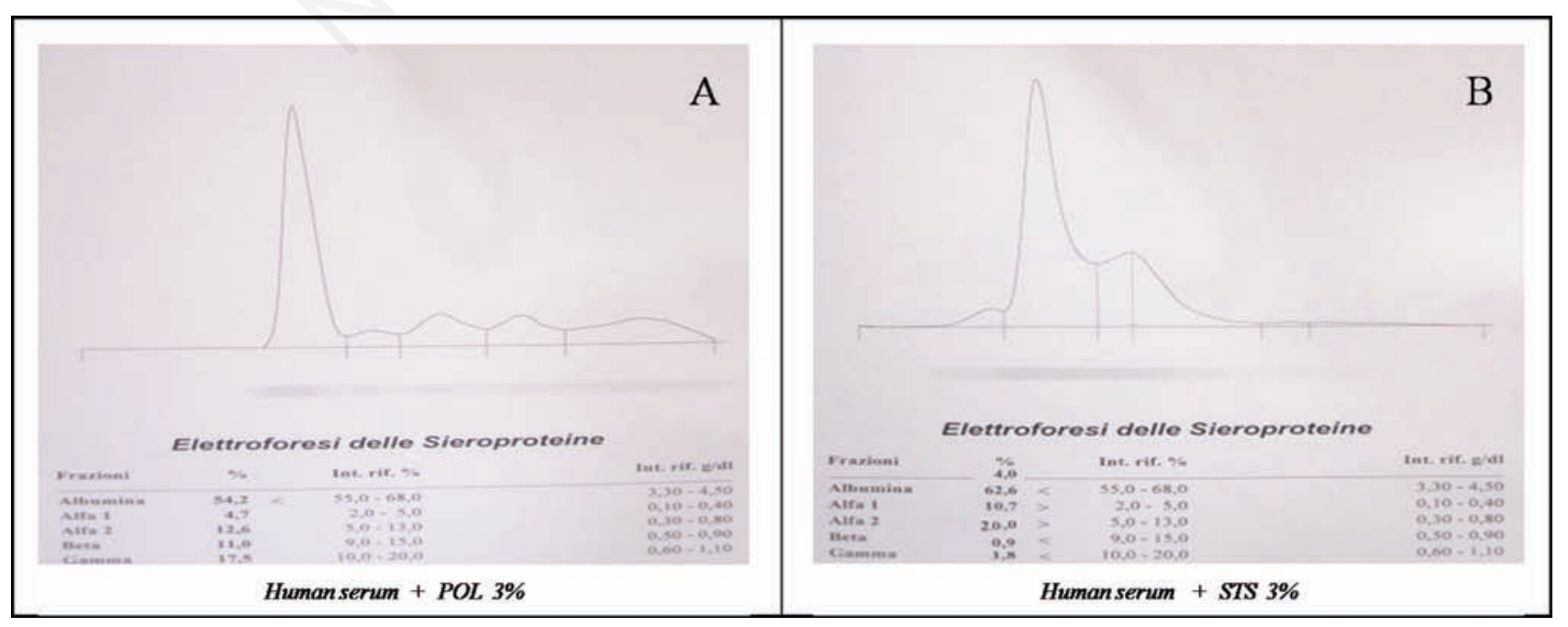

Figure 2. Electrophoretic runs of human blood with: A) polidocanol (POL) 3\%; and B) sodiumtetradecylsulphate (STS) 3\%. 
Both in the brachial and in the femoral vein fSTS was always 0 (Figure 5).

\section{Discussion}

Nowadays, FS is one of the most popular CVD therapeutic tools worldwide. ${ }^{1}$

Some articles have reported possible threatening side-effects. ${ }^{11}$

Nevertheless, recent reviews have demonstrated that FS complications and side effects remain uncommon, thus confirming FS efficacy and safety. ${ }^{12}$

On the other hand, the pathogenesis of FS-induced side effects still needs deeper investigation.

According to recent papers, the gas used to produce foam can be important in modification of the incidence of side effects, with a better safety profile for carbon dioxide-oxygen foam. ${ }^{8,9}$

But the side effects incidence isn't related only to the chosen gas. ${ }^{11,12}$

A deeper analysis of the biochemical interaction between the sclerosing agents and the blood components is mandatory to find out the possible new pathogenetic mechanisms of complications during sclerotherapy.

The present paper evaluates the sclerosing drug interaction with the human blood proteins, suggesting clinical consequences that improve the FS safety profile.

The first issue in the drug-protein binding assessment is identifying the exact timing in which it occurs.

A bias in the drug-protein CE assessed binding is the possible interaction between the two substances inside the test tube, during the same $\mathrm{CE}$ required processing time.

Therefore two different kind of electrophoresis have been used: CE and AGE.

$\mathrm{CE}$ is a more sophisticated analysis, designed to separate species and, based on their size, to charge ratio in the interior of a small capillary that is filled with an electrolyte. This characteristic brings a higher sensitivity, but requires some minutes to have the automatized process started.

To the contrary, AGE is a totally manual procedure, bringing less precise measurement, but providing the test result in a few seconds. ${ }^{13-20}$

The overlapping drug-protein peaks of $\mathrm{CE}$ and AGE demonstrated how the bond, and thus the inactivation, occurs after just a few seconds by the SA contact with the human blood.

Consequently, all the sclerosing drug circulation within the lungs is bounded to the proteins, and thus inactive.

Moreover, the FS safety profile is confirmed by the lack of detectible free sclerosing drug not only in the upper limb venous circulation, but also in the ipsilateral femoral vein.

Further considerations regarding coagula- tion modification were reported by Parsi last year, ${ }^{3}$ demonstrating how low concentration sclerosants inhibit platelet aggregation because of GPIIb/IIIa activation. The present study suggests that proteins like antiplasmin could be also involved. This is a $\alpha 2$ globulin which when bound to STS the lock on the plas- min is removed, thus enhancing fibrinolysis This may represent a possible rationale for fewer post-sclerotherapic deep venous thromboses with the STS use.

It has recently been suggested that FS complications and side effects may be related to new pathogenetic mechanisms, based on

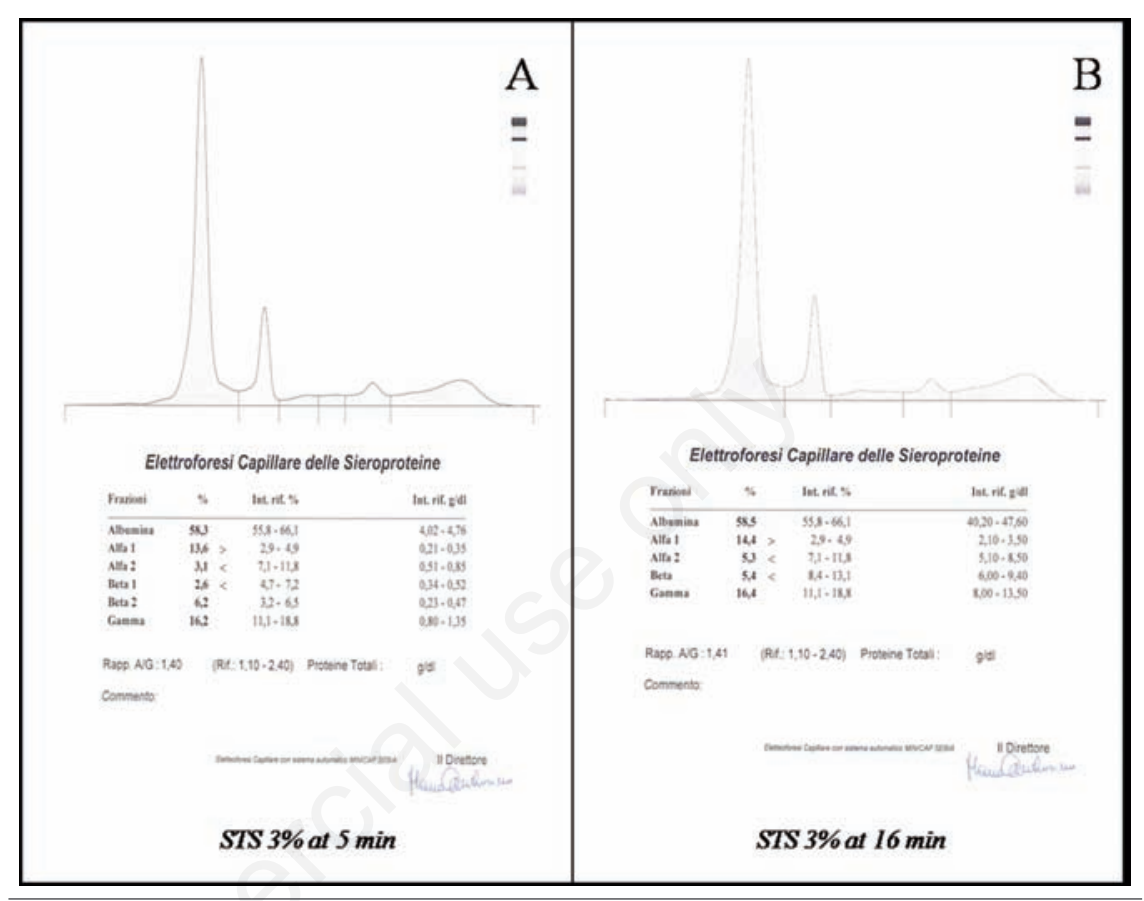

Figure 3. Sodiumtetradecylsulphate (STS) 3\% and blood electrophoretic run along the observation time at A) 5 and B) 16 min.

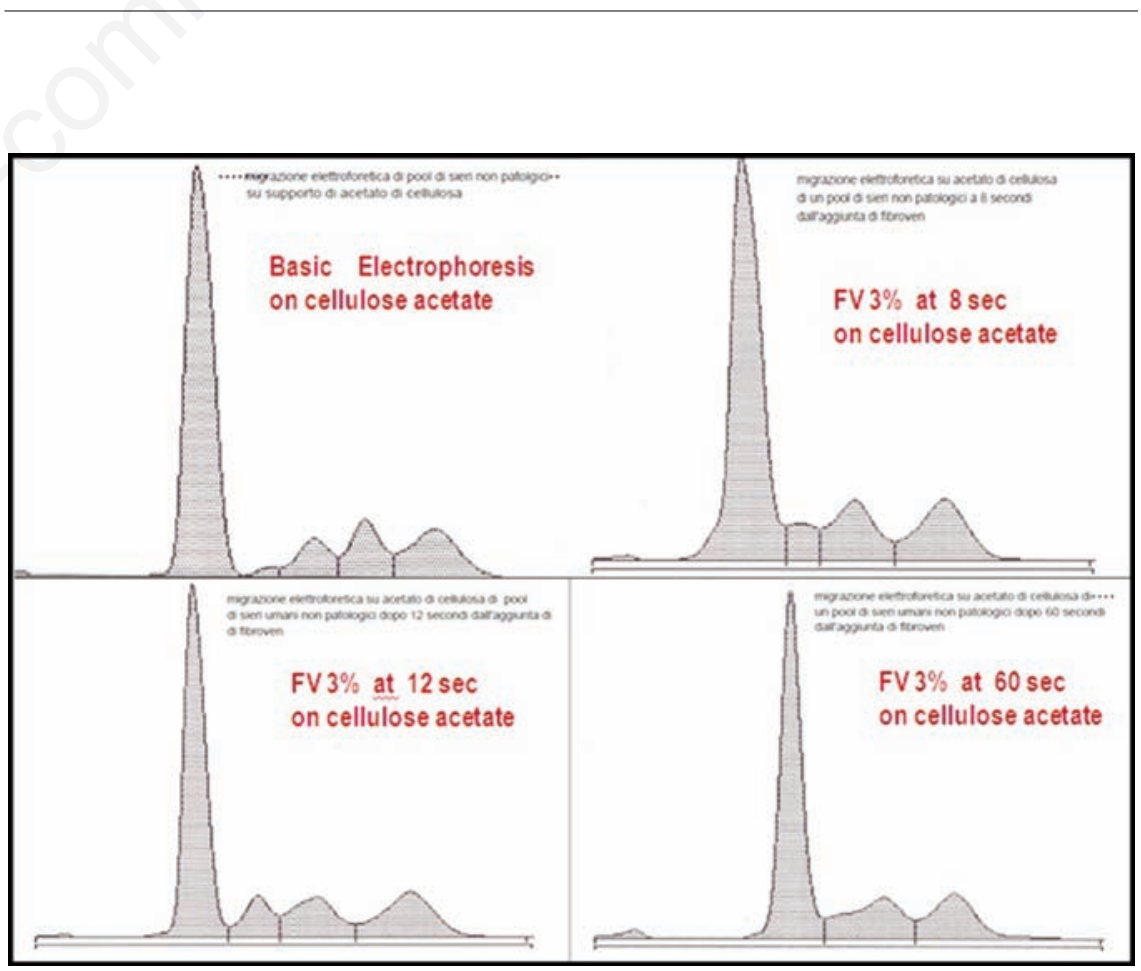

Figure 4. Sodiumtetradecylsulphate 3\% cellulose acetate electrophoretic run. 


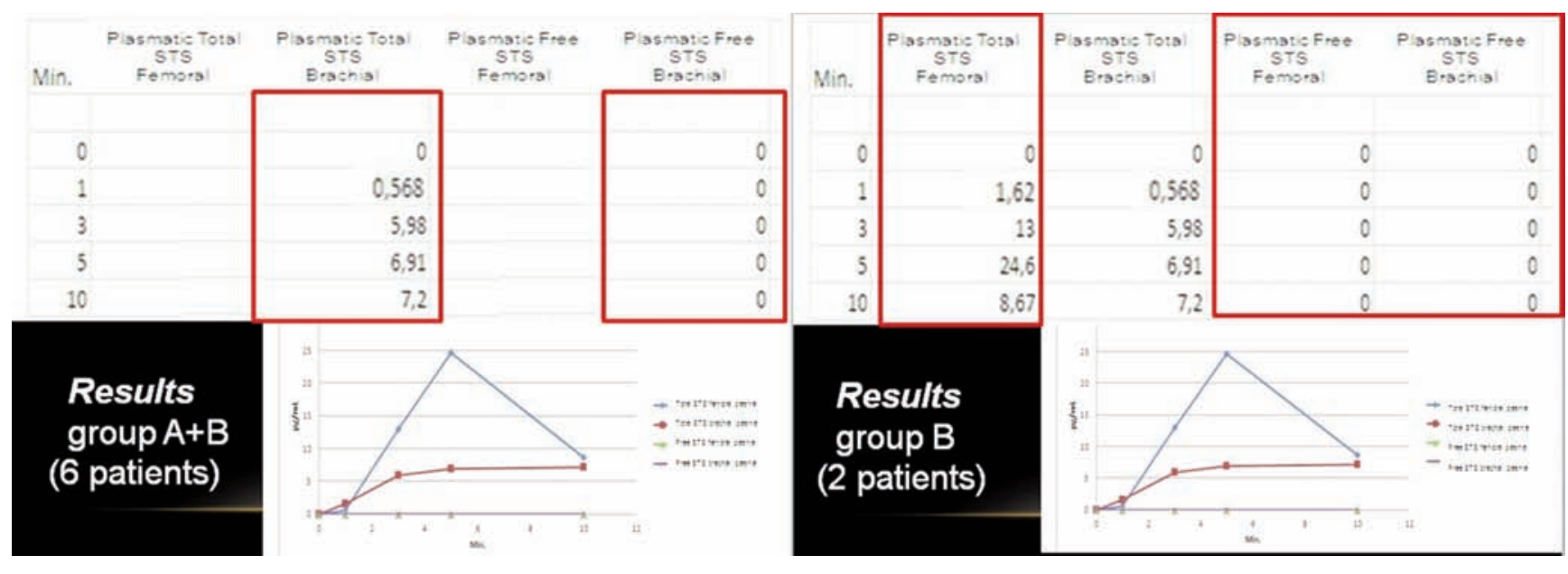

Figure 5. Assessment of free and bound sodiumtetradecylsulphate (STS) in the brachial and in the femoral vein.

endothelial release of vasoactive molecules, such as endothelin-1, histamine or serotonin. ${ }^{22-28}$

Further analysis should be aimed to elucidate both the post-sclerotherapic endothelial catabolite identification and deeper insight into the drug-protein kinetic.

\section{Conclusions}

Foam sclerotherapy safety remains a hot topic in the phlebology community, despite everyday clinical use which demonstrates an extremely low complication and side effect rate.

No data are available concerning the in vivo binding and neutralization of the $\mathrm{SA}$ whenever inside the blood stream.

The present paper offers two investigations demonstrating the SA binding features when in contact with human blood and the consequent kinetics within the venous network.

The results demonstrate in vivo the rapid inactivation of the SA in circulating blood, thus their high safety profile.

\section{References}

1. Murad MH, Coto-Yglesias F, ZumaetaGarcia M, et al. A systemic review and meta-analysis of the treatments of varicose veins. J Vasc Surg 2011;53:49S-65S.

2. Parsi K, Exner T, Connor DE, et al. In vitro effects of detergent sclerosants on coagulation, platelets and microparticles. Sur J Vasc Endovasc Surg 2007;34:731-40.

3. Parsi K, Connor DE, Pilotelle A, et al. Low concentration detergent sclerosants induce platelet activation but inhibit aggregation due to suppression of GpIIb/IIIa activation in vitro. Thromb Res 2012;130:472-8.

4. Parsi K, Exner T, Connor DE, et al. The lytic effects of detergent scerosants on erythrocytes, platelets, endothelial cells and microparticles are attenuated by albumin and other plasma components in vitro. Eur J Vasc Endovasc Surg 2008;36:216-23.

5. Watkins MR. Deactivation of sodium tetradecyl sulphate injection by blood proteins. Eur J Vasc Endovasc Surg 2011; 41:521-5.

6. Rathbun S, Norris A, Stoner J. Efficacy and safety of endovenous foam sclerotherapy: metan-analysis for treatment of venous disorders. Phlebology 2012;27:105-17.

7. Coleridge-Smith P. Sclerotherapy and foam sclerotherapy for varicose veins. Phlebology 2009;24:260-9.

8. Morrison N, Neuhardt DL, Rogers CR, et al. Incidence of side effects using carbon dioxide-oxygen foam for chemical ablation of superficial veins of the lower extremity. Eur J Vasc Endovasc Surg 2010;40:407-13.

9. Morrison N, Neuhardt DL, Rogers CR et al. Comparisons of side effects using air and carbon dioxide foam for endovenous chemical ablation. J Vasc Surg 2008;47: 830-6.

10. Beckitt T, Elstone A, Ashley S. Air versus physiological gas for ultrasound guided foam sclerotherapy treatment of varicose veins. Eur J Vasc Endovasc Surg 2011; 42:115.

11. Guex JJ. Complications and side-effects of foam sclerotherapy. Phlebology 2009;24: 270-4.

12. Guex JJ. Complications of sclerotherapy: an update. Dermatol Surg 2010;36:1056-63.
13. Merlini G, Marciano S, Gasparro C, et al. The Pavia approach to clinical protein analysis. Clin Chem Lab Med 2001;39: 1025-8.

14. Gay-Bellile C, Bengoufa D, Houze P, et al. Automated multicapillary electrophoresis for analysis of human serum proteins. Clin Chem 2003;49:1909-15.

15. Petersen JR, Okorodudu AO, Mohammed A, et al. Capillary electrophoresis and its application in the clinical laboratory. Clin Chim Acta 2003;330:1-30.

16. Bossuyt X. Advances in serum protein electrophoresis. Adv Clin Chem 2006;42:43-80.

17. Segura Carretero A, Cruces-Blanco C, Cortacero Ramírez S, et al. Application of micellar electrokinetic capillary chromatography to the analysis of uncharged pesticides of environmental impact. $\mathrm{J}$ Agric Food Chem 2004;52:5791-5.

18. Cavazza A, Corradini C, Lauria A, et al. Rapid analysis of essential and branchedchain amino acids in nutraceutical products by micellar electrokinetic capillary chromatography. J Agric Food Chem 2000; 48:3324-9.

19. Dorsey JG, Foley JP, Cooper WT, Barford RA, Barth HG. Liquid chromatography: theory and methodology. Anal Chem 1990; 62:324-56.

20. Rodrigues MR, Caramão EB, Arce L, et al. Determination of monoterpene hydrocarbons and alcohols in Majorana hortensis Moench by micellar electrokinetic capillary chromatographic. J Agric Food Chem 2002;50:4215-20.

21. Tessari L. Nouvelle technique d'obtention de la sclero-mousse. Phlebologie 1997; 53:129.

22. Dohonal J, Garvin J. The Interaction of Dodecyl and Tetradecyl Sulfate with pro- 
teins during Polyacrylamide gel electrophoresis. Biochim Biophys Acta 1979; 576 393-403.

23. Frullini A, Felice F, Burchielli S, et al. High production of endothelin after foam sclerotherapy: a new pathogenetic hypothesis for neurological and visual disturbances after sclerotherapy. Phlebology 2011;26:203-8.

24. Holm P. Endothelin in the pulmonary cir- culation with special reference to hypoxic pulmonary vasoconstriction. Scand Cardiovasc J Suppl 1997;46:1-40.

25. Lusher TF. Endothelin and endothelin antagonists: pharmacology and clinical implications. Agents Actions Suppl 1995; 45:237-53.

26. Ferrara F, Allaert FA, Ferrara G. Les médiateurs chimiques dans certaines complica- tions de la sclérothérapie. Phlébologie 2012;65:27-31.

27. Hu WW. Role of histamine and its receptors in cerebral ischemia. ACS Chem Neurosci 2012;3:238-47.

28. Frullini A, Barsotti MC, Santoni T, et al. Significant endothelin release in patients treated with foam sclerotherapy. Dermatol Surg 2012;38:74-7. 